\title{
BOUNDEDNESS AND INVARIANT METRICS FOR DIFFEOMORPHISM COCYCLES OVER HYPERBOLIC SYSTEMS
}

\author{
VICTORIA SADOVSKAYA*
}

\begin{abstract}
Let $\mathcal{A}$ be a Hölder continuous cocycle over a hyperbolic dynamical system with values in the group of diffeomorphisms of a compact manifold $\mathcal{M}$. We consider the periodic data of $\mathcal{A}$, i.e., the set of its return values along the periodic orbits in the base. We show that if the periodic data of $\mathcal{A}$ is bounded in $\operatorname{Diff}^{q}(\mathcal{M})$, $q>1$, then the set of values of the cocycle is bounded in $\operatorname{Diff}^{r}(\mathcal{M})$ for each $r<q$. Moreover, such a cocycle is isometric with respect to a Hölder continuous family of Riemannian metrics on $\mathcal{M}$.
\end{abstract}

\section{InTRODUCTION AND STATEMENT OF THE RESULtS}

Group-valued cocycles over hyperbolic systems have been extensively studied starting with the work of A. Livšic [Liv71, Liv72], who obtained definitive results for commutative groups and made some progress for more general ones. The subsequent research was focused on non-abelian groups, starting with compact groups and proceeding to more general ones, see [KtN] for a survey of results prior to 2008. The case of $G L(d, \mathbb{R})$ and Lie groups is now relatively well understood, see for example [PW01, dlLW10, Ka11, KaS10, S15]. Cocycles with values in diffeomorphism groups give another important and dynamically natural class, which is harder to analyze.

In this paper we consider the group $\operatorname{Diff}^{r}(\mathcal{M})$ of $C^{r}$ diffeomorphisms of a compact connected manifold $\mathcal{M}$ and study cocycles over a hyperbolic dynamical system with values in $\operatorname{Diff}^{r}(\mathcal{M})$. By a hyperbolic system we mean either a transitive Anosov diffeomorphism of a compact connected manifold, or a topologically mixing diffeomorphism of a locally maximal hyperbolic set, or a mixing subshift of finite type, see Section 2.1. We focus on the problem of obtaining information about the cocycle from its periodic data, that is, its return values along the periodic orbits in the base. This is one of the central questions for cocycles over hyperbolic systems. The basic problem in this area is to show that a cocycle with the identity periodic data is cohomologous to the identity cocycle. This was done in [NT95, dlLW10] under additional assumptions on growth of the cocycle and recently in [KP16, AKL18, Gu] just from the periodic data.

\footnotetext{
* Supported in part by NSF grant DMS-1764216.

Mathematical subject classification: 37D20, 54H15.

Keywords: Cocycle, diffeomorphism group, periodic orbit, hyperbolic system, symbolic system.
} 
In this paper we obtain results for much broader class of cocycles, which have uniformly bounded periodic data. We show that all iterates of such a cocycle remain uniformly bounded and the cocycle is, in a sense, a cocycle of isometries.

Definition 1.1. Let $f$ be a homeomorphism of a compact metric space $X$ and let $A$ be a function from $X$ to $\operatorname{Diff}^{r}(\mathcal{M})$. The $\operatorname{Diff}^{r}(\mathcal{M})$-valued cocycle over $f$ generated by $A$ is the map $\mathcal{A}: X \times \mathbb{Z} \rightarrow \operatorname{Diff}^{r}(\mathcal{M})$ defined by $\mathcal{A}(x, 0)=I d$ and for $n \in \mathbb{N}$,

$$
\mathcal{A}(x, n)=\mathcal{A}_{x}^{n}=A\left(f^{n-1} x\right) \circ \cdots \circ A(x) \text { and } \mathcal{A}(x,-n)=\mathcal{A}_{x}^{-n}=\left(\mathcal{A}_{f^{-n} x}^{n}\right)^{-1} .
$$

Clearly, $\mathcal{A}$ satisfies the cocycle equation $\mathcal{A}_{x}^{n+k}=\mathcal{A}_{f^{k} x}^{n} \circ \mathcal{A}_{x}^{k}$.

Cocycles can be considered in any regularity. We say that a cocycle $\mathcal{A}$ is bounded, continuous, or Hölder continuous if this property holds for its generator $A(x)=\mathcal{A}_{x}$. We call a set $S$ in $\operatorname{Diff}^{r}(\mathcal{M})$ bounded if $\|g\|_{C^{r}}$ and $\left\|g^{-1}\right\|_{C^{r}}$ are bounded uniformly in $g \in S$. Here $\|\cdot\|_{C^{r}}$ denotes the usual $C^{r}$ norm adapted to the manifold setting, see Section 2.2, where a distance $d_{C^{r}}$ between diffeomorphisms is also defined. A $\operatorname{Diff}^{r}(\mathcal{M})$-valued cocycle $\mathcal{A}$ is $\beta$-Hölder, $0<\beta \leq 1$, if there exists $c>0$ such that

$$
d_{C^{r}}\left(\mathcal{A}_{x}, \mathcal{A}_{y}\right) \leq c d_{X}(x, y)^{\beta} \text { for all } x, y \in X .
$$

Hölder continuity is the most natural setting for cocycles over hyperbolic systems, especially since we include non-smooth systems in the base.

For a cocycle $\mathcal{A}$, we consider the periodic data set $\mathcal{A}_{P}$ and the set of all values $\mathcal{A}_{X}$,

$$
\mathcal{A}_{P}=\left\{\mathcal{A}_{p}^{k}: p=f^{k} p, p \in X, k \in \mathbb{N}\right\} \quad \text { and } \quad \mathcal{A}_{X}=\left\{\mathcal{A}_{x}^{n}: x \in X, n \in \mathbb{Z}\right\} .
$$

Our main results are the following two theorems. The first one gives boundedness of the cocycle and the second one yields an invariant metric.

Theorem 1.2. Let $(X, f)$ be a hyperbolic system, let $\mathcal{M}$ be a compact connected manifold, let $k \in \mathbb{N}$, and let $\mathcal{A}$ be a bounded Diff ${ }^{k+1}(\mathcal{M})$-valued cocycle over $f$ which is Hölder continuous as a Diff $(\mathcal{M})$-valued cocycle with $q=k+\gamma, 0<\gamma<1$.

(i) If the periodic data set $\mathcal{A}_{P}$ is bounded in $\operatorname{Diff}^{q}(\mathcal{M})$, then the value set $\mathcal{A}_{X}$ is bounded in $\operatorname{Diff}^{r}(\mathcal{M})$ for any $r<q$.

(ii) If $\mathcal{A}_{P}$ is bounded in $\operatorname{Diff}^{1}(\mathcal{M})$, then $\mathcal{A}_{X}$ is also bounded in $\operatorname{Diff}^{1}(\mathcal{M})$.

Boundedness of cocycles allows, in particular, to obtain higher regularity of a continuous transfer map between them by the results in [NT96, [NT98.

Theorem 1.3. Let $(X, f)$ and $\mathcal{M}$ be as above, and let $\mathcal{A}$ be a bounded $\operatorname{Diff}^{2}(\mathcal{M})$ valued cocycle over $f$ that is $\beta$-Hölder continuous as a Diff $(\mathcal{M})$-valued cocycle with $q=1+\gamma, 0<\gamma<1$. If the periodic data set $\mathcal{A}_{P}$ is bounded in Diff' $(\mathcal{M})$, then there exists a family of Riemannian metrics $\left\{\tau_{x}: x \in X\right\}$ on $\mathcal{M}$ such that

$$
\mathcal{A}_{x}:\left(\mathcal{M}, \tau_{x}\right) \rightarrow\left(\mathcal{M}, \tau_{f x}\right) \quad \text { is an isometry for each } x \in X .
$$

Moreover, for any $\alpha<\gamma$ each $\tau_{x}$ is $\alpha$-Hölder continuous on $\mathcal{M}$ and depends Hölder continuously on $x$ in $C^{\alpha}$ distance with exponent $\beta(\gamma-\alpha)$. 
We note that uniform boundedness of the periodic data is crucial in our theorems. Indeed, S. Hurtado constructed in [H18] an example of Diff ${ }^{\infty}\left(S^{3} \times S^{1}\right)$-valued cocycles over a Bernoulli shift for which every periodic value $\mathcal{A}_{p}^{k}$ is an isometry of some $C^{\infty}$ Riemannian metric on $\mathcal{M}$, but the cocycle has positive exponential growth rate of derivatives. Also, our $C^{1+\text { Hölder }}$ regularity assumption for the cocycle is essential as it is known that an isometry of any $\alpha$-Hölder Riemannian metric is $C^{1+\alpha}$ [T06].

\section{PRELIMINARIES}

\subsection{Hyperbolic dynamical systems. See [KtH] for details.}

Transitive Anosov diffeomorphisms. A diffeomorphism $f$ of compact connected manifold $X$ is called Anosov if there exist a splitting of the tangent bundle $T X$ into a direct sum of two $D f$-invariant continuous subbundles $E^{s}$ and $E^{u}$, a Riemannian metric on $X$, and a number $\lambda$ such that

$$
\left\|D_{x} f\left(v^{s}\right)\right\|<\lambda<1<\lambda^{-1}<\left\|D_{x} f\left(v^{u}\right)\right\|
$$

for any $x \in X$ and unit vectors $v^{s} \in E^{s}(x)$ and $v^{u} \in E^{u}(x)$. The stable and unstable subbundles $E^{s}$ and $E^{u}$ are tangent to the stable and unstable foliations $W^{s}$ and $W^{u}$. The local stable manifold of $x, W_{l o c}^{s}(x)$, is a small ball in $W^{s}(x)$ centered at $x$ so that

$$
d_{X}\left(f^{n} x, f^{n} y\right) \leq \lambda^{n} d_{X}(x, y) \quad \text { for all } y \in W_{l o c}^{s}(x) \text { and } n \in \mathbb{N} .
$$

Local unstable manifolds are defined similarly. We assume that they are small enough so that $W_{l o c}^{s}(x) \cap W_{l o c}^{u}(z)$ consists of a single point for any sufficiently close $x, z \in X$.

A diffeomorphism is said to be (topologically) transitive if there is a point $x$ in $X$ with dense orbit. All known examples of Anosov diffeomorphisms have this property.

Mixing diffeomorphisms of locally maximal hyperbolic sets. More generally, let $f$ be a diffeomorphism of a manifold $\mathcal{N}$. A compact $f$-invariant set $X \subset \mathcal{N}$ is called hyperbolic if there exist a continuous $D f$-invariant splitting $T_{X} \mathcal{N}=E^{s} \oplus E^{u}$, and a Riemannian metric on an open set $U \supset X$ such that (2.1) holds for all $x \in X$. Local stable and unstable manifolds are defined similarly for any $x \in X$ and we denote their intersections with $X$ by $W_{l o c}^{s}(x)$ and $W_{l o c}^{y}(y)$. The set $X$ is called locally maximal if $X=\bigcap_{n \in \mathbb{Z}} f^{-n}(U)$ for some open set $U \supset X$. This property ensures that $W_{l o c}^{s}(x) \cap W_{l o c}^{y}(y)$ exists in $X$. The map $\left.f\right|_{X}$ is called topologically mixing if for any two open non-empty subsets $U, V$ of $X$ there is $N \in \mathbb{N}$ such that $f^{n}(U) \cap V \neq \emptyset$ for all $n \geq N$. Topological mixing implies transitivity.

Mixing subshifts of finite type. Let $M$ be $k \times k$ matrix with entries from $\{0,1\}$ such that all entries of $M^{N}$ are positive for some $N$. Let

$$
X=\left\{x=\left(x_{n}\right)_{n \in \mathbb{Z}}: 1 \leq x_{n} \leq k \text { and } M_{x_{n}, x_{n+1}}=1 \text { for every } n \in \mathbb{Z}\right\} .
$$

The shift map $f: X \rightarrow X$ is defined by $(f(x))_{n}=x_{n+1}$. The system $(X, f)$ is called a mixing subshift of finite type. We fix $\lambda \in(0,1)$ and consider the metric

$$
d(x, y)=d_{\lambda}(x, y)=\lambda^{n(x, y)}, \text { where } n(x, y)=\min \left\{|i|: x_{i} \neq y_{i}\right\} .
$$


The following sets play the role of the local stable and unstable manifolds of $x$

$$
W_{l o c}^{s}(x)=\left\{y: x_{i}=y_{i}, \quad i \geq 0\right\}, \quad W_{l o c}^{u}(x)=\left\{y: x_{i}=y_{i}, \quad i \leq 0\right\} .
$$

Indeed, for all $x \in X, y \in W_{l o c}^{s}(x), y^{\prime} \in W_{l o c}^{u}(x)$, and $n \in \mathbb{N}$,

$$
d\left(f^{n} x, f^{n} y\right)=\lambda^{n} d(x, y) \text { and } d\left(f^{-n} x, f^{-n} y\right)=\lambda^{n} d(x, y),
$$

and for any $x, z \in X$ with $d(x, z)<1$ the intersection of $W_{l o c}^{s}(x)$ and $W_{l o c}^{u}(z)$ consists of a single point, $y=\left(y_{n}\right)$ such that $y_{n}=x_{n}$ for $n \geq 0$ and $y_{n}=z_{n}$ for $n \leq 0$.

2.2. Distances on the space of diffeomorphisms. We fix a smooth background Riemannian metric and the corresponding distance $d_{\mathcal{M}}$ on $\mathcal{M}$. Recall that $\operatorname{Diff}^{r}(\mathcal{M})$, $r \geq 1$, is the set of $C^{r}$ diffeomorphisms of $\mathcal{M}$. The $C^{r}$ topology on $\operatorname{Diff}^{r}(\mathcal{M})$ can be defined using coordinate patches and the $C^{r}$ norm for the Euclidean space.

For diffeomorphisms $g$ and $h$ of $\mathcal{M}$ we set

$$
d_{C^{0}}(g, h)=\max _{t \in \mathcal{M}} d_{\mathcal{M}}(g(t), h(t))+\max _{t \in \mathcal{M}} d_{\mathcal{M}}\left(g^{-1}(t), h^{-1}(t)\right) .
$$

For any $g \in \operatorname{Diff}^{r}(\mathcal{M}), r \in \mathbb{N}$, we can define its $C^{r}$ size as

$$
|g|_{C^{r}}=\|g\|_{C^{r}}+\left\|g^{-1}\right\|_{C^{r}}, \quad \text { where }\|g\|_{C^{r}}=\max _{t \in \mathcal{M}} d_{\mathcal{M}}(g(t), t)+\max _{1 \leq i \leq r} \max _{t \in \mathcal{M}}\left\|D_{t}^{i} g\right\|,
$$

where $D_{t}^{i} g$ is the derivative of $g$ of order $i$ at $t$ and its norm is defined as the norm of the corresponding multilinear form from $T_{t} \mathcal{M}$ to $T_{g(t)} \mathcal{M}$ with respect the Riemannian metric. For $r=k+\alpha, k \in \mathbb{N}, 0<\alpha<1$, the definition is similar with

$$
\|g\|_{C^{r}}=\|g\|_{C^{k}}+\sup \left\{\left\|D_{t}^{k}-D_{t^{\prime}}^{k}\right\| \cdot d_{\mathcal{M}}\left(t, t^{\prime}\right)^{-\alpha}: t, t^{\prime} \in \mathcal{M}, 0<d_{\mathcal{M}}\left(t, t^{\prime}\right)<\epsilon_{0}\right\},
$$

where $\epsilon_{0}$ is chosen small compared to the injectivity radius of $\mathcal{M}$ so that the tangent bundle is locally trivialized via parallel transport and the difference makes sense.

We call a set $S$ bounded in $\operatorname{Diff}^{r}(\mathcal{M})$ if $\left\{|g|_{C^{r}}: g \in S\right\}$ is bounded.

A natural distance $d_{C^{r}}(g, h)$ on $\operatorname{Diff}^{r}(\mathcal{M}), r \in \mathbb{N}$, was defined in dlLW10 as the infimum of the lengths of piecewise $C^{1}$ paths in $\operatorname{Diff}^{r}(\mathcal{M})$ connecting $h$ with $g$ and $h^{-1}$ with $g^{-1}$, where the length of a path $p_{s}$ is

$$
\ell_{C^{r}}\left(p_{s}\right)=\max _{0 \leq i \leq r} \max _{t \in \mathcal{M}} \int\left\|\frac{d}{d s}\left(D_{t}^{i} p_{s}\right)\right\| d s
$$

see [dlLW10, Section 5] for details. For $r=k+\alpha$, one also adds the corresponding Hölder term:

$$
\begin{gathered}
\ell_{C^{r}}\left(p_{s}\right)=\ell_{C^{k}}\left(p_{s}\right)+\max _{t \in \mathcal{M}} \int\left|\frac{d}{d s}\left\|\left(D^{k} p_{s}\right)\right\|_{\alpha, t}\right| d s, \quad \text { where } \\
\left\|D^{k} g\right\|_{\alpha, t}=\sup \left\{\left\|D_{t^{\prime}}^{k}-D_{t}^{k}\right\| \cdot d_{\mathcal{M}}\left(t^{\prime}, t\right)^{-\alpha}: t^{\prime} \in \mathcal{M}, 0<d_{\mathcal{M}}\left(t, t^{\prime}\right)<\epsilon_{0}\right\} .
\end{gathered}
$$

We will consider distances only between sufficiently $C^{r}$-close diffeomorphisms, $r>1$. Then the distance $d_{C^{r}}(g, h)$ is Lipschitz equivalent to $\|g-h\|_{C^{r}}+\left\|g^{-1}-h^{-1}\right\|_{C^{r}}$, 
where the difference is understood using local trivialization. More specifically, there exist constants $\kappa$ and $\delta_{0}>0$ depending only on $r$ and the Riemannian metric so that

$$
\kappa^{-1} d_{C^{r}}(g, h) \leq\|g-h\|_{C^{r}}+\left\|g^{-1}-h^{-1}\right\|_{C^{r}} \leq \kappa d_{C^{r}}(g, h),
$$

provided that either $d_{C^{r}}(g, h)<\delta_{0}|g|_{C^{r}}^{-1}$ or $\|g-h\|_{C^{r}}+\left\|g^{-1}-h^{-1}\right\|_{C^{r}}<\delta_{0}|g|_{C^{r}}^{-1}$. The first inequality in (2.2) can be obtained using an interpolating path

$$
p_{s}(t)=\exp _{g(t)}\left(s \cdot \exp _{g(t)}^{-1} h(t)\right)
$$

between $C^{r}$-close diffeomorphisms. It is analogous to the "straight line homotopy" $g+s(h-g)$ with $\frac{d}{d s} p_{s}=h-g$. The $C^{r}$ closeness of $g$ and $h$ ensures that each $p_{s}$ is a diffeomorphism as its differential is invertible. The second inequality follows from the mean value theorem. When the diffeomorphisms are not necessarily close, we have a one-sided estimate

$$
|g|_{C^{r}} \leq \exp \left(\kappa^{\prime} d_{C^{0}}(g, h)\right) \cdot\left(|h|_{C^{r}}+d_{C^{r}}(g, h)\right) .
$$

where the constant $\kappa^{\prime}$ depends only on the Riemannian metric. It is given by dlLW10, Lemma 5.1] for $r \in \mathbb{N}$ and is obtained similarly for $r=k+\alpha$.

\section{Proof of Theorem 1.2}

3.1. Slow growth of the cocycle. First we show that the cocycle has slow growth using an extension of the following recent result by Avila, Kocsard, and Liu.

Theorem 3.1. AKL18, Theorem 2.5] Let $f: X \rightarrow X$ be a hyperbolic homeomorphism and let $\mathcal{A}: X \rightarrow \operatorname{Diff}^{1+\gamma}(\mathcal{M}), \gamma>0$, be a Hölder continuous cocycle.

If $\mathcal{A}_{P}=I d$, that is the cocycle has the identity periodic data, then

$$
\lim _{n \rightarrow \pm \infty} n^{-1} \log \left\|D_{t} \mathcal{A}_{x}^{n}\right\|=0 \text { for all }(x, t) \text { in } X \times \mathcal{M} .
$$

Proposition 3.2. The conclusion of Theorem 3.1 holds under the weaker assumption on the periodic data, that the set $\mathcal{A}_{P}$ is bounded in $\operatorname{Diff}^{1}(\mathcal{M})$.

Proof. This can be seen in the first three paragraphs of the Proof of Theorem 1.1 in Section 5 of [AKL18]. Indeed, assuming (3.1) does not hold, they show existence of a periodic point $p=f^{n} p$ in $X$ and $t \in \mathcal{M}$ for which some singular value of the derivative $D_{t} \mathcal{A}_{p}^{n}$ is arbitrarily large. This contradicts boundedness of $\mathcal{A}_{P}$ in $\operatorname{Diff}^{1}(\mathcal{M})$.

We consider the vector bundle $\mathcal{V}$ over $X \times \mathcal{M}$ with fiber $\mathcal{V}_{(x, t)}=T_{t} \mathcal{M}$ and the linear cocycle

$$
\mathcal{B}_{(x, t)}=D_{t} \mathcal{A}_{x} \text { on } \mathcal{V} \text { over the map } F(x, t)=\left(f x, \mathcal{A}_{x}(t)\right) .
$$

The iterates of $\mathcal{B}$ are given by $\mathcal{B}_{(x, t)}^{n}: T_{t} \mathcal{M} \rightarrow T_{\mathcal{A}_{x}^{n}(t)} \mathcal{M}$, where

$$
\mathcal{B}_{(x, t)}^{n}=D_{t} \mathcal{A}_{x}^{n}=D_{\mathcal{A}_{x}^{n-1}(t)} \mathcal{A}_{f^{n-1} x} \circ \ldots \circ D_{\mathcal{A}_{x}(t)} \mathcal{A}_{f x} \circ D_{t} \mathcal{A}_{x} .
$$

Since the periodic data set $\mathcal{A}_{P}$ is bounded in $\operatorname{Diff}^{q}(\mathcal{M})$ and hence in $\operatorname{Diff}^{1}(\mathcal{M})$, Proposition 3.2 applies and yields (3.1), which implies that all Lyapunov exponents 
of $\mathcal{B}_{(x, t)}$ are zero with respect to any $F$-invariant measure on $X \times \mathcal{M}$. It is well-known that the latter implies sub-exponential growth of the norm, see e.g. [Schr98]. More precisely, for each $\epsilon>0$ there exists $K_{\epsilon}$ such that for all $x \in X$,

$$
\sup \left\{\left\|D_{t} \mathcal{A}_{x}^{n}\right\|: t \in \mathcal{M}\right\} \leq K_{\epsilon} e^{|n| \epsilon} \quad \text { for all } n \in \mathbb{Z},
$$

where the case of $n<0$ follows from the similar estimates of the inverse of $\mathcal{A}$. Since the cocycle $\mathcal{A}_{x}$ is bounded in $C^{k+1}$, it follows that higher derivatives also grow subexponentially, see e.g. dILW10, Lemma 5.5]: for each $\epsilon>0$ there exists $K_{\epsilon}$ such that for all $x \in X$ and $1 \leq i \leq k+1$,

$$
\sup \left\{\left\|D_{t}^{i} \mathcal{A}_{x}^{n}\right\|: t \in \mathcal{M}\right\} \leq K_{\epsilon} e^{|n| \epsilon} \quad \text { for all } n \in \mathbb{Z} .
$$

Therefore, since $q \leq k+1$, for each $\epsilon>0$ there exists $K_{\epsilon}$ such that for all $x \in X$,

$$
\left|\mathcal{A}_{x}^{n}\right|_{C^{q}} \leq K_{\epsilon} e^{|n| \epsilon} \quad \text { for all } n \in \mathbb{Z}
$$

3.2. Holonomies. In this section we establish existence and regularity of holonomies for the cocycle $\mathcal{A}$. This is a fundamental property of cocycles with sufficiently slow growth. For $\operatorname{Diff}^{q}(\mathcal{M})$-valued cocycles with integer $q \geq 2$, the holonomies in $\operatorname{Diff}^{q-1}(\mathcal{M})$ were constructed in BK15] using estimates from in dlLW10. We consider an arbitrary $q>1$ and obtain holonomies in $\operatorname{Diff}^{r}(\mathcal{M})$ for any $r<q$. This allows us to obtain our main results with any $q>1$ and have an arbitrarily small loss of regularity. Using the results from BK15] in our arguments would result in the loss of several derivatives as in BK15, Theorem 1.1].

Our proof follows the usual approach of showing that $\left\{\left(\mathcal{A}_{y}^{n}\right)^{-1} \circ \mathcal{A}_{x}^{n}\right\}$ is a Cauchy sequence by estimating the distances between consecutive terms. The main difficulty for cocycles of diffeomorphisms is bounding the distortion of distances produced by composition on the left and on the right. These estimates are given in Lemmas 3.5 and 3.6 below. The main difference with [BK15] is that we work with non-integer $q$ and $r$ and obtain a distortion estimate (3.5) of Hölder rather than Lipschitz type. This allows us to loose arbitrarily small part of $q$, at the expense of obtaining lower Hölder regularity of the resulting holonomies along $W^{s}$ in (H3).

Proposition 3.3. Let $\mathcal{A}$ be a Diff $(\mathcal{M})$-valued cocycle over a hyperbolic dynamical system, where $q=k+\gamma$ with $k \in \mathbb{N}$ and $0<\gamma<1$. Let $r=k+\alpha$, where $0<\alpha<\gamma$. Suppose that $\mathcal{A}$ is $\beta$-Hölder in Diff $(\mathcal{M})$, and for each $\epsilon>0$ there exists $K_{\epsilon}$ such that

$$
\left|\mathcal{A}_{x}^{n}\right|_{C^{q}} \leq K_{\epsilon} e^{\epsilon n} \quad \text { for all } n \in \mathbb{N} \text {. }
$$

Then for any $x \in X$ and $y \in W^{s}(x)$, the limit

$$
H_{x, y}^{s}=H_{x, y}^{\mathcal{A}, s}=\lim _{n \rightarrow+\infty}\left(\mathcal{A}_{y}^{n}\right)^{-1} \circ \mathcal{A}_{x}^{n}
$$

exists in Diff ${ }^{r}(\mathcal{M})$ and satisfies

(H1) $H_{x, x}^{s}=I d$ and $H_{y, z}^{s} \circ H_{x, y}^{s}=H_{x, z}^{s}$, which imply $\left(H_{x, y}^{s}\right)^{-1}=H_{y, x}^{s}$;

(H2) $H_{x, y}^{s}=\left(\mathcal{A}_{y}^{n}\right)^{-1} \circ H_{f^{n} x, f^{n} y}^{s} \circ \mathcal{A}_{x}^{n}$ for all $n \in \mathbb{N}$; 
(H3) There exists a constant $c$ such that for all $x \in X$ and $y \in W_{l o c}^{s}(x)$,

$$
d_{C^{r}}\left(H_{x, y}^{s}, I d\right) \leq c d_{X}(x, y)^{\beta \rho}, \quad \text { where } \rho=q-r,
$$

moreover,

$$
d_{C^{r}}\left(\left(\mathcal{A}_{y}^{n}\right)^{-1} \circ \mathcal{A}_{x}^{n}, I d\right) \leq c d_{X}(x, y)^{\beta \rho} \quad \text { for all } n \in \mathbb{N} .
$$

The maps $H_{x, y}^{s}$ are called the stable holonomies of $\mathcal{A}$. It is convenient to view $H_{x, y}^{s}$ as a $C^{r}$ diffeomorphism from $\mathcal{M}_{x}$ to $\mathcal{M}_{y}$, i.e., from the fiber at $x$ to the fiber at $y$. The unstable holonomies are defined similarly: for $y \in W^{u}(x)$,

$$
H_{x, y}^{u}=H_{x, y}^{\mathcal{A}, u}=\lim _{n \rightarrow-\infty}\left(\mathcal{A}_{y}^{n}\right)^{-1} \circ \mathcal{A}_{x}^{n}
$$

Remark 3.4. For the conclusion to hold, we do not need (3.2) to be satisfied for each $\epsilon>0$, just for a sufficiently small one so that $e^{2 \epsilon(1+r)} \lambda^{\beta \rho}<1$, where $\lambda$ is as in (2.1).

First we give some estimates for distances and norms. Lemma 3.5 follows directly from Propositions 5.5 in dlLO98, where the estimates are obtained for compositions of smooth maps between open sets in Euclidean spaces.

Lemma 3.5. dlLO98] For any $r \geq 1$ there exist constants $M_{r}$ and $M_{r}^{\prime}$ such that for any $h, g \in C^{r}(\mathcal{M})$,

$$
\begin{aligned}
\|h \circ g\|_{C^{r}} & \leq M_{r}^{\prime}\left(1+\|g\|_{C^{1}}^{r-1}\right)\left(\|h\|_{C^{1}}\|g\|_{C^{r}}+\|h\|_{C^{r}}\|g\|_{C^{1}}\right)+\|h\|_{C^{0}} \leq \\
& \leq M_{r}\|h\|_{C^{r}}\left(1+\|g\|_{C^{r}}\right)^{r} .
\end{aligned}
$$

The next lemma relies on further results of dlLO98 which involve differences of functions. We apply these results to close diffeomorphisms using estimate (2.2), and so we need to ensure sufficient closeness of the diffeomorphisms $h_{1}$ and $h_{2}$, as well as that of the compositions $g \circ h_{1} \circ \tilde{g}$ and $g \circ h_{2} \circ \tilde{g}$.

Lemma 3.6. Let $q=k+\gamma, r=k+\alpha$, and $\rho=q-r$, where $k \in \mathbb{N}$ and $0 \leq \alpha<\gamma \leq 1$. There exists a constant $M=M(r, q, \mathcal{M}, K)$ such that for any $g, \tilde{g} \in \operatorname{Diff}^{q}(\mathcal{M})$ and $h_{1}, h_{2} \in \operatorname{Diff}^{r}(\mathcal{M})$ with $\left|h_{1}\right|_{C^{r}},\left|h_{2}\right|_{C^{r}} \leq K$,

$$
\begin{aligned}
& d_{C^{r}}\left(g \circ h_{1} \circ \tilde{g}, g \circ h_{2} \circ \tilde{g}\right) \leq \\
& \leq M\left(\|g\|_{C^{q}}\left(1+\|\tilde{g}\|_{C^{r}}\right)^{r}+\left\|\tilde{g}^{-1}\right\|_{C^{q}}\left(1+\left\|g^{-1}\right\|_{C^{r}}\right)^{r}\right) \cdot d_{C^{r}}\left(h_{1}, h_{2}\right)^{\rho}
\end{aligned}
$$

provided that $d_{C^{r}}\left(h_{1}, h_{2}\right) \leq \delta_{0}\left|h_{1}\right|_{C^{r}}^{-1}$ and the right hand side of (3.5) is less than

$$
\delta_{0}\left(M_{r}^{2}\left(1+\left|h_{1}\right|_{C^{r}}\right)^{r}\left(\|g\|_{C^{r}}\left(1+\|\tilde{g}\|_{C^{r}}\right)^{r}+\left\|\tilde{g}^{-1}\right\|_{C^{r}}\left(1+\left\|g^{-1}\right\|_{C^{r}}\right)^{r}\right)\right)^{-1}
$$

where $\delta_{0}$ is as in (2.2).

Proof. We use (2.2) to estimate the distance between close diffeomorphisms together with the following estimate from [lLO98, Propositions 6.2(iii)]:

$$
\left\|g \circ h_{1}-g \circ h_{2}\right\|_{C^{r}} \leq M^{\prime}\|g\|_{C^{q}}\left\|h_{1}-h_{2}\right\|_{C^{r}}^{\rho},
$$


where $M^{\prime}=M^{\prime}(r, q, \mathcal{M}, K)$. Using this and Lemma 3.5 we obtain

$$
\begin{aligned}
& \left\|g \circ h_{1} \circ \tilde{g}-g \circ h_{2} \circ \tilde{g}\right\|_{C^{r}}=\left\|\left(g \circ h_{1}-g \circ h_{2}\right) \circ \tilde{g}\right\|_{C^{r}} \leq \\
& \leq M_{r}\left\|g \circ h_{1}-g \circ h_{2}\right\|_{C^{r}} \cdot\left(1+\|\tilde{g}\|_{C^{r}}\right)^{r} \leq \\
& \leq M_{r} M^{\prime}\|g\|_{C^{q}}\left\|h_{1}-h_{2}\right\|_{C^{r}}^{\rho} \cdot\left(1+\|\tilde{g}\|_{C^{r}}\right)^{r}=M^{\prime \prime}\|g\|_{C^{q}}\left(1+\|\tilde{g}\|_{C^{r}}\right)^{r} \cdot\left\|h_{1}-h_{2}\right\|_{C^{r}}^{\rho}
\end{aligned}
$$

It follows that

$$
\begin{aligned}
& \left\|g \circ h_{1} \circ \tilde{g}-g \circ h_{2} \circ \tilde{g}\right\|_{C^{r}}+\left\|\tilde{g}^{-1} \circ h_{1}^{-1} \circ g^{-1}-\tilde{g}^{-1} \circ h_{2}^{-1} \circ g^{-1}\right\|_{C^{r}} \leq \\
& \leq M^{\prime \prime}\|g\|_{C^{q}}\left(1+\|\tilde{g}\|_{C^{r}}\right)^{r} \cdot\left\|h_{1}-h_{2}\right\|_{C^{r}}^{\rho}+ \\
& \quad+M^{\prime \prime}\left\|\tilde{g}^{-1}\right\|_{C^{q}}\left(1+\left\|g^{-1}\right\|_{C^{r}}\right)^{r} \cdot\left\|h_{1}^{-1}-h_{2}^{-1}\right\|_{C^{r}}^{\rho} \leq \\
& \leq M^{\prime \prime}\left(\|g\|_{C^{q}}\left(1+\|\tilde{g}\|_{C^{r}}\right)^{r}+\left\|\tilde{g}^{-1}\right\|_{C^{q}}\left(1+\left\|g^{-1}\right\|_{C^{r}}\right)^{r}\right) \cdot \kappa d_{C^{r}}\left(h_{1}, h_{2}\right)^{\rho} .
\end{aligned}
$$

By (2.2), this yields the same estimate for $\kappa^{-1} d_{C^{r}}\left(g \circ h_{1} \circ \tilde{g}, g \circ h_{2} \circ \tilde{g}\right)$ and gives (3.5) with $M=M^{\prime \prime} \kappa^{2}$, provided that this estimate is at most $\delta_{0} \cdot\left|g \circ h_{1} \circ \tilde{g}\right|_{C^{r}}^{-1}$. The latter follows from the last assumption of the lemma and the following estimate for $\left|g \circ h_{1} \circ \tilde{g}\right|_{C^{r}}$. Applying Lemma 3.5 twice, we get

$$
\begin{aligned}
& \left\|g \circ h_{1} \circ \tilde{g}\right\|_{C^{r}}=\left\|\left(g \circ h_{1}\right) \circ \tilde{g}\right\|_{C^{r}} \leq M_{r}\left\|g \circ h_{1}\right\|_{C^{r}}\left(1+\|\tilde{g}\|_{C^{r}}\right)^{r} \leq \\
& \leq M_{r}^{2}\|g\|_{C^{r}}\left(1+\left\|h_{1}\right\|_{C^{r}}\right)^{r}\left(1+\|\tilde{g}\|_{C^{r}}\right)^{r} .
\end{aligned}
$$

Similarly,

$$
\left\|\left(g \circ h_{1} \circ \tilde{g}\right)^{-1}\right\|_{C^{r}} \leq M_{r}^{2}\left\|\tilde{g}^{-1}\right\|_{C^{r}}\left(1+\left\|h_{1}^{-1}\right\|_{C^{r}}\right)^{r}\left(1+\left\|g^{-1}\right\|_{C^{r}}\right)^{r},
$$

and hence

$$
\left|g \circ h_{1} \circ \tilde{g}\right|_{C^{r}} \leq M_{r}^{2}\left(1+\left|h_{1}\right|_{C^{r}}\right)^{r}\left(\|g\|_{C^{r}}\left(1+\|\tilde{g}\|_{C^{r}}\right)^{r}+\left\|\tilde{g}^{-1}\right\|_{C^{r}}\left(1+\left\|g^{-1}\right\|_{C^{r}}\right)^{r}\right) .
$$

Proof of Proposition 3.3. We fix $x \in X$ and construct $H_{x, y}^{s}$ for any $y \in W_{l o c}^{s}(x)$ sufficiently close to $x$. Then the map $H^{s}$ can be extended to the whole leaf $W^{s}(x)$ by the invariance property (H2).

For $y \in W_{l o c}^{s}(x)$, we have $d_{X}\left(f^{n} x, f^{n} y\right) \leq d_{X}(x, y) \lambda^{n}$ for all $n \in \mathbb{N}$, where $0<\lambda<1$ is as in (2.1). We consider the sequence of diffeomorphisms $\left\{\left(\mathcal{A}_{y}^{n}\right)^{-1} \circ \mathcal{A}_{x}^{n}\right\}$ and show that it is Cauchy in $\operatorname{Diff}^{r}(\mathcal{M})$.

We apply Lemma [3.6 with $h_{1}=$ Id and $h_{2}=\left(\mathcal{A}_{f^{n} y}\right)^{-1} \circ \mathcal{A}_{f^{n} x}$ to estimate the distance between consecutive terms. We will verify assumption (3.6) later.

$$
\begin{aligned}
& d_{C^{r}}\left(\left(\mathcal{A}_{y}^{n}\right)^{-1} \circ \mathcal{A}_{x}^{n},\left(\mathcal{A}_{y}^{n+1}\right)^{-1} \circ \mathcal{A}_{x}^{n+1}\right)= \\
& =d_{C^{r}}\left(\left(\mathcal{A}_{y}^{n}\right)^{-1} \circ \operatorname{Id} \circ \mathcal{A}_{x}^{n},\left(\mathcal{A}_{y}^{n}\right)^{-1} \circ\left(\mathcal{A}_{f^{n} y}\right)^{-1} \circ \mathcal{A}_{f^{n} x} \circ \mathcal{A}_{x}^{n}\right) \leq \\
& \leq M\left(\left\|\left(\mathcal{A}_{y}^{n}\right)^{-1}\right\|_{C^{q}}\left(1+\left\|\mathcal{A}_{x}^{n}\right\|_{C^{r}}\right)^{r}\right. \\
& \left.+\left\|\left(\mathcal{A}_{x}^{n}\right)^{-1}\right\|_{C^{q}}\left(1+\left\|\mathcal{A}_{y}^{n}\right\|_{C^{r}}\right)^{r}\right) \times \\
& \times d_{C^{r}}\left(\mathrm{Id},\left(\mathcal{A}_{f^{n} y}\right)^{-1} \circ \mathcal{A}_{f^{n} x}\right)^{\rho} .
\end{aligned}
$$


Using condition (3.2) we estimate

$$
\left\|\left(\mathcal{A}_{y}^{n}\right)^{-1}\right\|_{C^{q}}\left(1+\left\|\mathcal{A}_{x}^{n}\right\|_{C^{r}}\right)^{r} \leq K_{\epsilon} e^{\epsilon n}\left(1+K_{\epsilon} e^{\epsilon n}\right)^{r} \leq K_{\epsilon}^{\prime} e^{\epsilon n(1+r)} .
$$

Thus we have

$$
\begin{aligned}
& d_{C^{r}}\left(\left(\mathcal{A}_{y}^{n}\right)^{-1} \circ \mathcal{A}_{x}^{n},\left(\mathcal{A}_{y}^{n+1}\right)^{-1} \circ \mathcal{A}_{x}^{n+1}\right) \leq K_{\epsilon}^{\prime \prime} e^{\epsilon n(1+r)} \cdot \operatorname{dist}\left(f^{n} y, f^{n} x\right)^{\beta \rho} \leq \\
& \leq K_{\epsilon}^{\prime \prime} e^{\epsilon n(1+r)} \cdot\left(d_{X}(x, y) \lambda^{n}\right)^{\beta \rho}=K_{\epsilon}^{\prime \prime} d_{X}(x, y)^{\beta \rho} \cdot \theta^{n} \text {, where } \theta=e^{\epsilon(1+r)} \lambda^{\beta \rho} .
\end{aligned}
$$

To verify assumption (3.6), we similarly estimate

$$
\left\|\left(\mathcal{A}_{y}^{n}\right)^{-1}\right\|_{C^{r}}\left(1+\left\|\mathcal{A}_{x}^{n}\right\|_{C^{r}}\right)^{r}+\left\|\left(\mathcal{A}_{x}^{n}\right)^{-1}\right\|_{C^{r}}\left(1+\left\|\mathcal{A}_{y}^{n}\right\|_{C^{r}}\right)^{r} \leq 2 K_{\epsilon}^{\prime} e^{\epsilon n(1+r)} .
$$

We take $\epsilon>0$ such that $\theta e^{\epsilon(1+r)}<1$, and in particular $\theta<1$. Since

$$
K_{\epsilon}^{\prime \prime} d_{X}(x, y)^{\beta \rho} \cdot \theta^{n}<K_{\epsilon}^{\prime \prime} d_{X}(x, y)^{\beta \rho} \cdot e^{-n \epsilon(1+r)},
$$

we conclude that (3.6) is satisfied of all $n \in \mathbb{N}$ provided that $d_{X}(x, y)$ is small enough.

Therefore, by (3.7), $\left\{\left(\mathcal{A}_{y}^{n}\right)^{-1} \circ \mathcal{A}_{x}^{n}\right\}$ is a Cauchy sequence in $\operatorname{Diff}^{r}(\mathcal{M})$, and so it has a limit there. Properties (H1) and (H2) are easy to verify. Properties (H3) and (3.4) are obtained as follows. For every $n \in \mathbb{N}$ we have

$$
\begin{aligned}
& \left.d_{C^{r}}\left(\left(\mathcal{A}_{y}^{n}\right)^{-1} \circ \mathcal{A}_{x}^{n}, \mathrm{Id}\right)=d_{C^{r}}\left(\left(\mathcal{A}_{y}^{n}\right)^{-1} \circ \mathcal{A}_{x}^{n},\left(\mathcal{A}_{y}^{0}\right)^{-1} \circ \mathcal{A}_{x}^{0}\right)\right) \leq \\
& \leq \sum_{k=0}^{n-1} d_{C^{r}}\left(\left(\mathcal{A}_{y}^{k}\right)^{-1} \circ \mathcal{A}_{x}^{k},\left(\mathcal{A}_{y}^{k+1}\right)^{-1} \circ \mathcal{A}_{x}^{k+1}\right) \leq K_{\epsilon}^{\prime \prime} d_{X}(x, y)^{\beta \rho} \sum_{n=0}^{\infty} \theta^{n} \leq c d_{X}(x, y)^{\beta \rho} .
\end{aligned}
$$

Taking the limit as $n \rightarrow \infty$ we obtain $d_{C^{r}}\left(H_{x, y}^{s}\right.$, Id $) \leq c d_{X}(x, y)^{\beta \rho}$.

3.3. Boundedness of the cocycle. We assume that the periodic data set bounded in $|\cdot|_{C^{q}}$ in Proposition 3.7 and bounded in $|\cdot|_{C^{1}}$ in Proposition 3.9.

Proposition 3.7. Suppose that a cocycle $\mathcal{A}$ satisfies the assumptions of Proposition 3.3 and its periodic data set $\mathcal{A}_{P}$ is bounded in Diff $(\mathcal{M})$, where $q=k+\gamma, k \in \mathbb{N}$, $0<\gamma<1$. Then its value set $\mathcal{A}_{X}$ is bounded in $\operatorname{Diff}^{r}(\mathcal{M})$ for any $r<q$.

Proof. The base systems that we are considering satisfy the following closing property.

Lemma 3.8. (Anosov Closing Lemma [KtH, 6.4.15-17]) Let $(X, f)$ be a topologically mixing diffeomorphism of a locally maximal hyperbolic set. Then there exist constants $c, \delta^{\prime}>0$ such that for any $x \in X$ and $k \in \mathbb{N}$ with $\operatorname{dist}\left(x, f^{k} x\right)<\delta^{\prime}$ there exists a periodic point $p \in X$ with $f^{k} p=p$ such that the orbit segments $x, f x, \ldots, f^{k} x$ and $p, f p, \ldots, f^{k} p$ satisfy

$$
d_{X}\left(f^{i} x, f^{i} p\right) \leq c d_{X}\left(x, f^{k} x\right) \text { for every } i=0, \ldots, k .
$$

For subshifts of finite type this property can be observed directly.

Since the map $f$ is transitive, we can consider a point $z \in X$ whose orbit $O(z)=$ $\left\{f^{n} z: n \in \mathbb{Z}\right\}$ is dense in $X$. Let $f^{n_{1}} z$ and $f^{n_{2}} z$ be two points of $O(z)$ with 
$\delta:=\operatorname{dist}\left(f^{n_{1}} z, f^{n_{2}} z\right)<\delta^{\prime}$, where $\delta^{\prime}$ is as in Lemma 3.8. We assume that $n_{1}<n_{2}$ and denote

$$
w=f^{n_{1}} z \quad \text { and } \quad k=n_{2}-n_{1}, \quad \text { so that } \delta=\operatorname{dist}\left(w, f^{k} w\right)<\delta^{\prime} .
$$

Then there exists $p \in X$ with $f^{k} p=p$ such that $\operatorname{dist}\left(f^{i} w, f^{i} p\right) \leq c \delta$ for $i=0, \ldots, k$.

We fix $\epsilon>0$ and take $r_{1}=q-\epsilon$. Let $y$ be the point of intersection of $W_{l o c}^{s}(p)$ and $W_{\text {loc }}^{u}(w)$. Then by Proposition 3.3 (H3) there exists a constant $c_{1}$ independent of $p$ and $y$ such that

$$
d_{C^{r_{1}}}\left(\left(\mathcal{A}_{p}^{k}\right)^{-1} \circ \mathcal{A}_{y}^{k}, \mathrm{Id}\right) \leq c_{1} \delta^{\beta \epsilon}
$$

We use Lemma 3.6 with $g=\mathcal{A}_{p}^{k}, h_{1}=\left(\mathcal{A}_{p}^{k}\right)^{-1} \circ \mathcal{A}_{y}^{k}$, and $h_{1}=\mathrm{Id}=\tilde{g}$. Since the set $\mathcal{A}_{P}$ is bounded in $\operatorname{Diff}^{q}(\mathcal{M})$, all norms in (3.5) and (3.6) are similarly uniformly bounded and, in particular, condition (3.6) is satisfied if $\delta^{\prime}$ is chosen small enough. We conclude that

$$
d_{C^{r_{1}}}\left(\mathcal{A}_{y}^{k}, \mathcal{A}_{p}^{k}\right) \leq c\left(\left|\mathcal{A}_{p}^{k}\right|_{C^{r}}\right) \cdot d_{C^{r_{1}}}\left(\left(\mathcal{A}_{p}^{k}\right)^{-1} \circ \mathcal{A}_{y}^{k}, \mathrm{Id}\right)^{\epsilon} \leq c_{1} \delta^{\beta \epsilon^{2}} .
$$

Thus the set $\left\{\left|\mathcal{A}_{y}^{k}\right|_{C^{r_{1}}}\right\}$ is bounded by (2.3). A similar argument, using unstable holonomies, shows that the set $\left\{\left|A_{w}^{k}\right|_{C^{r_{2}}}\right\}$ is bounded for $r_{2}=r_{1}-\epsilon=q-2 \epsilon$.

Therefore, there exists a constant $c_{2}$ such that whenever $\delta:=\operatorname{dist}\left(f^{n_{1}} z, f^{n_{2}} z\right)<\delta^{\prime}$ with $k=n_{2}-n_{1}>0$, we have $\left|\mathcal{A}_{f^{n_{1}}}^{k}\right|_{C^{r_{2}}}<c_{2}$.

We take $m \in \mathbb{N}$ such that the set $\left\{f^{j} z:|j| \leq m\right\}$ is $\delta^{\prime}$-dense in $X$ and set

$$
c_{m}=\max \left\{\left|\mathcal{A}_{z}^{j}\right|_{C^{r_{2}}}:|j| \leq m\right\} .
$$

Let $n>m$. Then there exists $j$ with $|j| \leq m$, such that $d_{X}\left(f^{n} z, f^{j} z\right) \leq \delta^{\prime}$. Using Lemma 3.5, we obtain that

$$
\begin{aligned}
\left\|\mathcal{A}_{z}^{n}\right\|_{C^{r_{2}}} & =\left\|\mathcal{A}_{f^{j} z}^{n-j} \circ \mathcal{A}_{z}^{j}\right\|_{C^{r_{2}}} \leq M_{r_{2}}\left(1+\left\|\mathcal{A}_{z}^{j}\right\|_{C^{r_{2}}}\right)^{r_{2}}\left\|\mathcal{A}_{f^{j} z}^{n-j}\right\|_{C^{r_{2}}} \leq \\
& \leq M_{r_{2}}\left(1+c_{m}\right)^{r_{2}} \cdot c_{2} \leq c_{3} .
\end{aligned}
$$

The cases of $\left\{\left\|\left(\mathcal{A}_{z}^{n}\right)^{-1}\right\|_{C^{r_{2}}}\right\}$ and of $n<-m$ are similar. Thus there exists a constant $c$ such that

$$
\left|\mathcal{A}_{z}^{n}\right|_{C^{r_{2}}} \leq c \text { for all } n \in \mathbb{Z} \text {. }
$$

Since $O(z)$ is dense in $X$ and $\left|\mathcal{A}_{z}^{n}\right|_{C^{r_{2}}}$ is continuous on $X$ for each $n$, we conclude that $\left|\mathcal{A}_{x}^{n}\right|_{C^{r_{2}}}$ is uniformly bounded in $x \in X$ and $n \in \mathbb{Z}$. Since $\epsilon$ is arbitrary, the proposition follows.

Proposition 3.9. Suppose that a cocycle $\mathcal{A}$ satisfies the assumptions of Proposition 3.3 with $q=1+\gamma, \gamma>0$, and its periodic data set $\mathcal{A}_{P}$ is bounded in Diff ${ }^{1}(\mathcal{M})$. Then its value set $\mathcal{A}_{X}$ is also bounded in $\operatorname{Diff}^{1}(\mathcal{M})$.

Since $\mathcal{A}_{X}$ is bounded in $|\cdot|_{C^{0}}$, it suffices to show that the first derivatives are uniformly bounded. As in the previous proof, we consider the points $z, w, p$, and 
$y=W_{l o c}^{s}(p) \cap W_{l o c}^{u}(w)$. Then by (3.4) there exists a constant $c$ independent of $p$ and $y$ such that

$$
d_{C^{1}}\left(\left(\mathcal{A}_{p}^{k}\right)^{-1} \circ \mathcal{A}_{y}^{k}, \mathrm{Id}\right) \leq c \quad \text { and hence } \quad\left\|D_{t}\left(\left(\mathcal{A}_{p}^{k}\right)^{-1} \circ \mathcal{A}_{y}^{k}\right)^{ \pm 1}\right\| \leq c^{\prime}
$$

for some $c^{\prime}$. Since $\mathcal{A}_{y}^{k}=\mathcal{A}_{p}^{k} \circ\left(\left(\mathcal{A}_{p}^{k}\right)^{-1} \circ \mathcal{A}_{y}^{k}\right)$ and the set $\mathcal{A}_{P}$ is bounded in $|\cdot|_{C^{1}}$, we conclude that $\left\|D_{t}\left(\mathcal{A}_{y}^{k}\right)^{ \pm 1}\right\|$ is bounded uniformly in $t, k$, and $y$, as above, and thus the set $\left\{\mathcal{A}_{y}^{k}\right\}$ is bounded in $|\cdot|_{C^{1}}$. Then a similar argument shows that the set $\left\{\mathcal{A}_{w}^{k}\right\}$ is also bounded in $|\cdot|_{C^{1}}$. Therefore, there exists a constant $c^{\prime \prime}$ such that whenever $\operatorname{dist}\left(f^{n_{1}} z, f^{n_{2}} z\right)<\delta^{\prime}$ with $k=n_{2}-n_{1}>0$, we have $\left|\mathcal{A}_{f^{n}{ }_{1}}^{k}\right|_{C^{1}}<c^{\prime \prime}$.

We take $m \in \mathbb{N}$ such that the set $\left\{f^{j} z:|j| \leq m\right\}$ is $\delta^{\prime}$-dense in $X$ and let

$$
c_{m}=\max \left\{\left|\mathcal{A}_{z}^{m}\right|_{C^{1}}:|j| \leq m\right\} .
$$

Since for any $n>m$ there exists $j,|j| \leq m$, such that $d_{X}\left(f^{n} z, f^{j} z\right) \leq \delta^{\prime}$, we have

$$
\left\|D_{t}\left(\mathcal{A}_{z}^{n}\right)\right\|=\left\|D_{\mathcal{A}^{n-j}(t)}\left(\mathcal{A}_{z}^{j}\right) \circ D_{t}\left(\mathcal{A}_{f^{j} z}^{n-j}\right)\right\| \leq\left|\mathcal{A}_{z}^{j}\right|_{C^{1}} \cdot\left|\mathcal{A}_{f^{j} z}^{n-j}\right|_{C^{1}} \leq c_{m} c^{\prime \prime}
$$

and similarly for $\left\|D_{t}\left(\mathcal{A}_{z}^{n}\right)^{-1}\right\|$ and for $n<-m$. Thus the set $\left\{\left|\mathcal{A}_{z}^{n}\right|_{C^{1}}: n \in \mathbb{Z}\right\}$ is bounded, and it follows that $\mathcal{A}_{X}$ is bounded $|\cdot|_{C^{1}}$.

This completes the proof of Theorem 1.2 .

\section{Proof of Theorem 1.3}

We recall that $q=1+\gamma$, where $0<\gamma<1$. We fix $0<\alpha<\gamma$ and set $r=1+\alpha$. Since the periodic data set $\mathcal{A}_{P}$ is bounded in $\operatorname{Diff}^{q}(\mathcal{M})$, the cocycle $\mathcal{A}$ satisfies the conclusion of Proposition 3.3, and in particular has stable and unstable holonomies in $\operatorname{Diff}^{r}(\mathcal{M})$. Also, the set of values $\mathcal{A}_{X}$ is bounded in $\operatorname{Diff}^{r}(\mathcal{M})$ by Proposition 3.7.

As in Section 3.1, we consider the linear cocycle $\mathcal{B}_{(x, t)}=D_{t} \mathcal{A}_{x}$ over the map $F(x, t)=\left(f(x), \mathcal{A}_{x}(t)\right)$ on the vector bundle $\mathcal{V}$ over $X \times \mathcal{M}$ with fiber $\mathcal{V}_{(x, t)}=T_{t} \mathcal{M}$

The Proof of Theorem 1.3 is organized as follows. In Section 4.1 we construct an everywhere defined bounded measurable family $\hat{\tau}$ of inner products on the vector bundle $\mathcal{V}$ invariant under the cocycle $\mathcal{B}$. In Section 4.2 we show that $\hat{\tau}$ is Hölder continuous along each fiber $\mathcal{M}_{x}$ in $X \times \mathcal{M}$. In Section 4.4 we establish essential invariance of $\hat{\tau}$ under the holonomies of the linear cocycle $\mathcal{B}$ along the stable and unstable sets in the skew product. In Section 4.5, we consider a natural invariant measure $\hat{\mu}$ for the skew product that projects to the measure of maximal entropy $\mu$ for $X$. Then using the above essential invariance with respect to $\hat{\mu}$ we show that $\hat{\tau}_{x}$, the restriction of $\hat{\tau}$ to $\mathcal{M}_{x}$, is $\mu$-essentially invariant under the stable and unstable holonomies of the cocycle $\mathcal{A}$, as a Riemannian metric on the whole fiber $\mathcal{M}_{x}$. This yields essential Hölder continuity of $\hat{\tau}_{x}$ along the stable and unstable leaves in $X$, and hence global Hölder continuity.

The arguments in Sections 4.1 and 4.4 are similar to those in [S05, KaS10, KS13] for conformality of linear cocycles over hyperbolic and partially hyperbolic systems. However, the skew product $F$ is not a volume preserving smooth partially hyperbolic 
system as in [KS13, so we adapt arguments to our case. More importantly, $F$ is not accessible and so we need arguments in Section 4.2 to show regularity along the "center" $\mathcal{M}$ and in Section 4.5 to deduce global regularity.

\subsection{An invariant bounded measurable family $\hat{\tau}$ of inner products on $\mathcal{V}$.}

In this section, it suffices to assume that the cocycle $\mathcal{A}$ is bounded in $|\cdot|_{C^{1}}$, and hence $\left\|\mathcal{B}_{(x, t)}^{n}\right\|$ is uniformly bounded in $(x, t) \in X \times \mathcal{M}$ and $n \in \mathbb{Z}$.

The space $\mathcal{T}^{m}$ of inner products on $\mathbb{R}^{m}$ identifies with the space of real symmetric positive definite $m \times m$ matrices, which is isomorphic to $G L(m, \mathbb{R}) / S O(m, \mathbb{R})$. The group $G L(m, \mathbb{R})$ acts transitively on $\mathcal{T}^{m}$ via $A[E]=A^{T} E A$, where $A \in G L(m, \mathbb{R})$ and $E \in \mathcal{T}^{m}$. The space $\mathcal{T}^{m}$ is a Riemannian symmetric space of non-positive curvature when equipped with a certain $G L(m, \mathbb{R})$-invariant metric [La, Ch. XII, Theorem 1.2]. Using the background Riemannian metric on $\mathcal{V}$, we can identify an inner product with a symmetric linear operator. For each $(x, t) \in X \times \mathcal{M}$, we denote the space of inner products on $\mathcal{V}_{(x, t)}$ by $\mathcal{T}_{(x, t)}$, and so we obtain a bundle $\mathcal{T}$ over $X \times \mathcal{M}$ with fiber $\mathcal{T}_{(x, t)}$. We equip the fibers of $\mathcal{T}$ with the Riemannian metric mentioned above. We call a continuous (Hölder continuous, measurable) section of $\mathcal{T}$ a continuous (Hölder continuous, measurable) Riemannian metric on $\mathcal{V}$. A metric metric $\tau$ is called bounded if the distance between $\tau_{(x, t)}$ and $\tilde{\tau}_{(x, t)}$ is uniformly bounded on $X \times \mathcal{M}$ for a continuous metric $\tilde{\tau}$ on $\mathcal{V}$. For the linear cocycle $\mathcal{B}$, the pullback of an inner product $\tau_{F(x, t)}$ on $\mathcal{V}_{F(x, t)}$ to $\mathcal{V}_{(x, t)}$ is given by

$$
\left(\mathcal{B}_{(x, t)}^{*}\left(\tau_{F(x, t)}\right)\right)\left(v_{1}, v_{2}\right)=\tau_{F(x, t)}\left(\mathcal{B}_{z}\left(v_{1}\right),\left(\mathcal{B}_{z}\right) v_{2}\right) \quad \text { for } v_{1}, v_{2} \in \mathcal{V}_{(x, t)} .
$$

We say that a metric $\tau$ is $\mathcal{B}$-invariant if $\mathcal{B}^{*}(\tau)=\tau$.

Let $\tau$ be a continuous metric on $\mathcal{V}$. We consider the set

$$
S(x, t)=\left\{\left(\mathcal{B}_{(x, t)}^{n}\right)^{*}\left(\tau_{F^{n}(x, t)}\right): n \in \mathbb{Z}\right\} \subset \mathcal{T}_{(x, t)} .
$$

Since the cocycle $\mathcal{B}$ is bounded, the sets $S(x, t)$ have uniformly bounded diameters. Since the space $\mathcal{T}_{(x, t)}$ has non-positive curvature, for every $(x, t)$ there exists a unique smallest closed ball containing $S(x, t)$ [La, Ch. XI, Theorem 3.1]. We denote its center by $\hat{\tau}_{(x, t)}$. By the construction, the metric $\hat{\tau}$ is invariant under $\mathcal{B}$.

For any $k \geq 0$, the set

$$
S^{k}(x, t)=\left\{\left(\mathcal{B}_{(x, t)}^{n}\right)^{*}\left(\tau_{F^{n}(x, t)}\right):|n| \leq k\right\}
$$

depends continuously on $(x, t)$ in Hausdorff distance, and so does the center $\tau_{(x, t)}^{k}$ of the smallest ball containing $S^{k}(x, t)$ by Lemma 4.1 below. Since $S^{k}(x, t) \rightarrow S(x, t)$ in Hausdorff distance as $k \rightarrow \infty$ for any $(x, t)$, the metric $\hat{\tau}$ is the pointwise limit of continuous metrics $\tau_{(x, t)}^{k}$, and hence $\hat{\tau}$ is Borel measurable.

Lemma 4.1. Let $S_{1}$ and $S_{2}$ be bounded sets in $\mathcal{T}^{m}$ and let $B\left(c_{1}, r_{1}\right)$ and $B\left(c_{2}, r_{2}\right)$ be the smallest closed balls containing $S_{1}$ and $S_{2}$, respectively. Then $d_{\mathcal{T}^{m}}\left(c_{1}, c_{2}\right) \leq$ $d_{H}\left(S_{1}, S_{2}\right)$, where $d_{H}$ is the Hausdorff distance. 
Proof. Suppose $d_{\mathrm{H}}\left(S_{1}, S_{2}\right)<\epsilon$. Then, since $S_{1} \subset B\left(c_{1}, r_{1}\right)$, we have $S_{2} \subset B\left(c_{1}, r_{1}+\epsilon\right)$, and hence $B\left(c_{2}, r_{2}\right) \subset B\left(c_{1}, r_{1}+\epsilon\right)$ by minimality. Similarly, $B\left(c_{1}, r_{1}\right) \subset B\left(c_{2}, r_{2}+\epsilon\right)$.

If $d_{\mathcal{T} m}\left(c_{1}, c_{2}\right)>\epsilon$, we obtain a contradiction. Indeed, suppose $r_{2} \geq r_{1}$. Any two points in $\mathcal{T}^{m}$ lie on a unique geodesic, which is isometric to $\mathbb{R}$. Therefore there exists a point $c$ on the geodesic through $c_{1}$ and $c_{2}$ with

$$
d_{\mathcal{T}^{m}}\left(c, c_{2}\right)=r_{2} \text { and } d_{\mathcal{T}^{m}}\left(c, c_{1}\right)=d_{\mathcal{T}^{m}}\left(c, c_{2}\right)+d_{\mathcal{T}^{m}}\left(c_{2}, c_{1}\right)>r_{2}+\epsilon \geq r_{1}+\epsilon .
$$

This point is in $B\left(c_{2}, r_{2}\right)$, but not in $B\left(c_{1}, r_{1}+\epsilon\right)$. Thus $\operatorname{dist}\left(c_{1}, c_{2}\right) \leq \epsilon$.

4.2. Hölder continuity of $\hat{\tau}$ along the fibers $\mathcal{M}_{x}$ in $X \times \mathcal{M}$. We recall that by Proposition 3.7 the value set $\mathcal{A}_{X}$ is bounded in $\operatorname{Diff}^{r}(\mathcal{M})$, where $r=1+\alpha$.

We start with an $\alpha$-Hölder continuous metric $\tau$ on $\mathcal{V}$ and obtain a bounded Borel measurable metric $\hat{\tau}$, as in the previous section. Below we show that $\hat{\tau}$ is Hölder along the fibers. We denote by $\hat{\tau}_{x}$ the restriction of the metric $\hat{\tau}$ to the fiber $\mathcal{M}_{x}$.

Proposition 4.2. For each $x \in X$ the metric $\hat{\tau}_{x}$ is $\alpha$-Hölder continuous on $\mathcal{M}_{x}$, more precisely, there exists a constant $c$ such that

$$
d_{\mathcal{T}}\left(\hat{\tau}(x, t), \hat{\tau}\left(x, t^{\prime}\right)\right) \leq c d_{\mathcal{M}}\left(t, t^{\prime}\right)^{\alpha} \quad \text { for all } x \in X \text { and } t, t^{\prime} \in \mathcal{M} .
$$

Proof. We use the following lemma. It was proven in [KaS10] for conformal structures rather than inner products, but the proof works without significant modifications.

Lemma 4.3. ( $c f$. KaS10, Lemma 4.5]) Let $\tau$ be an inner product on $\mathbb{R}^{m}$ and $B$ be a linear transformation of $\mathbb{R}^{m}$ sufficiently close to the identity. Then

$$
d_{\mathcal{T}}(\tau, B(\tau)) \leq c_{1}(\tau) \cdot\|B-I d\|,
$$

where the function $c_{1}(\tau)$ is bounded on compact sets in $\mathcal{T}^{m}$.

In the chain of inequalities below, we use the following: the pullback action is an isometry; the metric $\tau$ is $\alpha$-Hölder continuous and in particular bounded; Lemma 4.3. and the fact that since $\mathcal{A}_{X}$ is bounded in $|\cdot|_{C^{1+\alpha}}$, the norm $\left\|\left(\mathcal{B}_{(x, t)}^{n}\right)^{ \pm 1}\right\|$ is bounded and there is a constant $c_{2}$ such that

$$
\left\|\mathcal{B}_{(x, t)}^{n}-\mathcal{B}_{\left(x, t^{\prime}\right)}^{n}\right\| \leq c_{2} d_{\mathcal{M}}\left(t, t^{\prime}\right)^{\alpha} .
$$

For each $n \in \mathbb{Z}$ we have

$$
\begin{aligned}
& d_{\mathcal{T}}\left(\left(\mathcal{B}_{(x, t)}^{n}\right)^{*}\left(\tau_{F^{n}(x, t)}\right),\left(\mathcal{B}_{\left(x, t^{\prime}\right)}^{n}\right)^{*}\left(\tau_{F^{n}\left(x, t^{\prime}\right)}\right)\right) \leq \\
& \leq d_{\mathcal{T}}\left(\left(\mathcal{B}_{(x, t)}^{n}\right)^{*}\left(\tau_{F^{n}(x, t)}\right),\left(\mathcal{B}_{(x, t)}^{n}\right)^{*}\left(\tau_{F^{n}\left(x, t^{\prime}\right)}\right)\right)+ \\
& \quad \quad+d_{\mathcal{T}}\left(\left(\mathcal{B}_{(x, t)}^{n}\right)^{*}\left(\tau_{F^{n}\left(x, t^{\prime}\right)}\right),\left(\mathcal{B}_{\left(x, t^{\prime}\right)}^{n}\right)^{*}\left(\tau_{F^{n}\left(x, t^{\prime}\right)}\right)\right) \leq \\
& \left.\leq d_{\mathcal{T}}\left(\tau_{F^{n}(x, t)}, \tau_{F^{n}\left(x, t^{\prime}\right)}\right)+d_{\mathcal{T}}\left(\tau_{F^{n}\left(x, t^{\prime}\right)},\left(\mathcal{B}_{(x, t)}^{n}\right)^{-1} \mathcal{B}_{\left(x, t^{\prime}\right)}^{n}\right)^{*}\left(\tau_{F^{n}\left(x, t^{\prime}\right)}\right)\right) \\
& \leq c_{3} d_{\mathcal{M}}\left(t, t^{\prime}\right)^{\alpha}+c_{1}\left(\tau_{F^{n}\left(x, t^{\prime}\right)}\right)\left\|\left(\mathcal{B}_{(x, t)}^{n}\right)^{-1} \mathcal{B}_{\left(x, t^{\prime}\right)}^{n}-\mathrm{Id}\right\| \leq \\
& \leq c_{3} d_{\mathcal{M}}\left(t, t^{\prime}\right)^{\alpha}+c_{4}\left\|\left(\mathcal{B}_{(x, t)}^{n}\right)^{-1}\right\| \cdot\left\|\mathcal{B}_{(x, t)}^{n}-\mathcal{B}_{\left(x, t^{\prime}\right)}^{n}\right\| \leq \\
& \leq c_{3} d_{\mathcal{M}}\left(t, t^{\prime}\right)^{\alpha}+c_{4} c_{5} c_{2} d_{\mathcal{M}}\left(t, t^{\prime}\right)^{\alpha}=c_{6} d_{\mathcal{M}}\left(t, t^{\prime}\right)^{\alpha} .
\end{aligned}
$$


Thus for each $n$,

$$
d_{\mathcal{T}}\left(\left(\mathcal{B}_{(x, t)}^{n}\right)^{*}\left(\tau_{F^{n}(x, t)}\right),\left(\mathcal{B}_{\left(x, t^{\prime}\right)}^{n}\right)^{*}\left(\tau_{F^{n}\left(x, t^{\prime}\right)}\right)\right) \leq c d_{\mathcal{M}}\left(t, t^{\prime}\right)^{\alpha} \quad \text { for all } t, t^{\prime} \in \mathcal{M}
$$

where the constant $c=c_{6}$ is independent of $x$ and $n$. It follows immediately that the Hausdorff distance between the sets $S^{k}(x, t)$ and $S^{k}\left(x, t^{\prime}\right)$ given by (4.1) satisfies

$$
d_{\mathrm{H}}\left(S^{k}(x, t), S^{k}\left(x, t^{\prime}\right)\right) \leq c d_{\mathcal{M}}\left(t, t^{\prime}\right)^{\alpha} \quad \text { for all } x \in X \text { and } t, t^{\prime} \in \mathcal{M} .
$$

Hence by Lemma 4.1 the center $\tau_{(x, t)}^{k}$ of the smallest closed ball containing $S^{k}(x, t)$ also satisfies

$$
d_{\mathcal{T}}\left(\tau_{(x, t)}^{k}, \tau_{\left(x, t^{\prime}\right)}^{k}\right) \leq c d_{\mathcal{M}}\left(t, t^{\prime}\right)^{\alpha} \quad \text { for all } x, k, t, t^{\prime} .
$$

Passing to the limit as $k \rightarrow \infty$, we obtain (4.2).

4.3. Stable and unstable sets for $F$. We consider the map $F(x, t)=\left(f(x), \mathcal{A}_{x}(t)\right)$ of the set $X \times \mathcal{M}$. While it is not partially hyperbolic in the classical sense, we can define the stable sets $\tilde{W}^{s}$ for $F$ using the stable holonomies $H_{x, y}^{s}$ given by Proposition 3.3. The unstable sets $\tilde{W}^{u}$ are defined similarly. For any $(x, t) \in X \times \mathcal{M}$, we set

$$
\tilde{W}^{s}(x, t)=\left\{\left(y, t^{\prime}\right) \in X \times \mathcal{M}: y \in W^{s}(x), t^{\prime}=H_{x, y}^{s}(t)\right\} .
$$

We will only use the following contraction property for these sets:

$$
d_{X \times \mathcal{M}}\left(F^{n}(x, t), F^{n}\left(y, t^{\prime}\right)\right) \rightarrow 0 \quad \text { as } n \rightarrow \infty
$$

for any $(x, t) \in X \times \mathcal{M}$ and $\left(y, t^{\prime}\right) \in \tilde{W}^{s}(x, t)$. It holds since

$$
\begin{aligned}
& d_{X \times \mathcal{M}}\left(F^{n}(x, t), F^{n}\left(y, t^{\prime}\right)\right)=d_{X \times \mathcal{M}}\left(\left(f^{n} x, \mathcal{A}_{x}^{n}(t)\right),\left(f^{n} y, \mathcal{A}_{y}^{n}\left(t^{\prime}\right)\right)\right)= \\
& =d_{X}\left(f^{n} x, f^{n} y\right)+d_{\mathcal{M}}\left(\mathcal{A}_{x}^{n}(t), \mathcal{A}_{y}^{n}\left(t^{\prime}\right)\right) \rightarrow 0 \quad \text { as } n \rightarrow \infty
\end{aligned}
$$

as $d_{X}\left(f^{n} x, f^{n} y\right) \rightarrow 0$ and

$$
\mathcal{A}_{y}^{n}\left(t^{\prime}\right)=H_{f^{n} x, f^{n} y}^{s} \circ \mathcal{A}_{x}^{n} \circ H_{y, x}^{s}\left(t^{\prime}\right)=H_{f^{n} x, f^{n} y}^{s} \circ \mathcal{A}_{x}^{n}(t),
$$

where $d_{C^{r}}\left(H_{f^{n} x, f^{n} y}^{s}, \mathrm{Id}\right) \leq c d_{X}\left(f^{n} x, f^{n} y\right)^{\beta \rho} \rightarrow 0$ by (H3) in Proposition 3.3 .

4.4. Essential invariance of $\hat{\tau}$ under the holonomies. For convenience, in the remaining two sections we will use the push forward of an inner product by a linear map, which is defined as the pull-back by its inverse: $L_{*}=\left(L^{-1}\right)^{*}$.

First we show that $\hat{\tau}$ is essentially invariant under the derivatives of $H^{s}$ along the stable sets of $F$ in $X \times \mathcal{M}$. These derivatives can be interpreted as stable holonomies of the cocycle $\mathcal{B}$. The statement and proof for the unstable holonomies are similar.

Proposition 4.4. Let $\nu$ be an ergodic $F$-invariant probability measure on $X \times \mathcal{M}$. If $\tau$ is a $\nu$-measurable $\mathcal{B}$-invariant metric on $\mathcal{V}$, then $\tau$ is essentially $H^{s}$-invariant, more precisely, there exists an F-invariant set $E \subset X \times \mathcal{M}$ with $\nu(E)=1$ such that

$$
\hat{\tau}\left(y, t^{\prime}\right)=\left(D_{t} H_{x, y}^{s}\right)_{*}(\hat{\tau}(x, t)) \quad \text { for all }(x, t),\left(y, t^{\prime}\right) \in E \quad \text { with }\left(y, t^{\prime}\right) \in \tilde{W}_{l o c}^{s}(x, t) .
$$


Proof. To simplify the notations, we write $\tau$ for $\hat{\tau}$ and $d$ for $d_{\mathcal{T}}$, and for $y \in W_{l o c}^{s}(x)$, $t \in \mathcal{M}_{x}$, and $t^{\prime}=H_{x, y}^{s}(t) \in \mathcal{M}_{y}$, we set

$$
z=(x, t), \quad z^{\prime}=\left(y, t^{\prime}\right) \text { so } z^{\prime} \in \tilde{W}_{l o c}(z), \quad z_{n}=F^{n}(z), \text { and } z_{n}^{\prime}=F^{n}\left(z^{\prime}\right) .
$$

Since $\tau$ is $\nu$-measurable, by Lusin's Theorem there exists a compact set $S \subset X \times \mathcal{M}$ with $\nu(S)>1 / 2$ so that $\tau$ is uniformly continuous and hence bounded on $S$. Let $E$ be the set of points in $X \times \mathcal{M}$ for which the asymptotic frequency of visiting $S$ equals $\nu(S)>1 / 2$. By Birkhoff Ergodic Theorem, $\nu(E)=1$.

Suppose that both $z$ and $z^{\prime}$ are in $E$. We will show that

$$
d\left(\tau\left(z^{\prime}\right),\left(D_{t} H_{x y}^{s}\right)_{*}(\tau(z))\right)=0 \text {, that is, } \tau\left(z^{\prime}\right)=\left(D_{t} H_{x y}^{s}\right)_{*}(\tau(z)) .
$$

Property (H2) of the holonomies, $H_{x, y}^{s}=\left(\mathcal{A}_{y}^{n}\right)^{-1} \circ H_{f^{n} x, f^{n} y}^{s} \circ \mathcal{A}_{x}^{n}$, implies

$$
\begin{aligned}
& D_{t} H_{x, y}^{s}=D_{\mathcal{A}_{y}^{n}\left(t^{\prime}\right)}\left(\mathcal{A}_{y}^{n}\right)^{-1} \circ D_{\mathcal{A}_{x}^{n}(t)} H_{f^{n} x, f^{n} y}^{s} \circ D_{t} \mathcal{A}_{x}^{n}= \\
& =\left(\mathcal{B}_{\left(y, t^{\prime}\right)}^{n}\right)^{-1} \circ D_{\mathcal{A}_{x}^{n}(t)} H_{f^{n} x, f^{n} y}^{s} \circ \mathcal{B}_{(x, t)}^{n}=\left(\mathcal{B}_{z^{\prime}}^{n}\right)^{-1} \circ D_{\mathcal{A}_{x}^{n}(t)} H_{f^{n} x, f^{n} y}^{s} \circ \mathcal{B}_{z}^{n} .
\end{aligned}
$$

Since the metric $\tau$ is invariant under the cocycle $\mathcal{B}$, and $\mathcal{B}$ induces an isometry on the space of inner products, we have

$$
\begin{aligned}
& d\left(\tau\left(z^{\prime}\right),\left(D_{t} H_{x y}^{s}\right)_{*}(\tau(z))\right)= \\
& =d\left(\tau\left(z^{\prime}\right),\left(\left(\mathcal{B}_{z^{\prime}}^{n}\right)^{-1}\right)_{*}\left(D_{\mathcal{A}_{x}^{n}(t)} H_{f^{n} x, f^{n} y}^{s} \circ \mathcal{B}_{z}^{n}\right)_{*}(\tau(z))\right)= \\
& =d\left(\left(\mathcal{B}_{z^{\prime}}^{n}\right)_{*}\left(\tau\left(z^{\prime}\right)\right),\left(D_{\mathcal{A}_{x}^{n}(t)} H_{f^{n} x, f^{n} y}^{s}\right)_{*}\left(\mathcal{B}_{z}^{n}\right)_{*}(\tau(z))\right)= \\
& =d\left(\tau\left(z_{n}^{\prime}\right),\left(D_{\mathcal{A}_{x}^{n}(t)} H_{f^{n} x, f^{n} y}^{s}\right)_{*}\left(\tau\left(z_{n}\right)\right)\right)= \\
& \leq d\left(\tau\left(z_{n}^{\prime}\right), \tau\left(z_{n}\right)\right)+d\left(\tau\left(z_{n}\right),\left(D_{\mathcal{A}_{x}^{n}(t)} H_{f^{n} x, f^{n} y}^{s}\right)_{*}\left(\tau\left(z_{n}\right)\right)\right) .
\end{aligned}
$$

Since $z, z^{\prime} \in E$, there exists a sequence $\left\{n_{i}\right\}$ such that both $z_{n_{i}}$ and $z_{n_{i}}^{\prime}$ are in $S$ for each $i$. Since $z^{\prime} \in \tilde{W}_{l o c}^{s}(z), d_{X \times \mathcal{M}}\left(z_{n_{i}}, z_{n_{i}}^{\prime}\right) \rightarrow 0$ and hence $\left.d\left(\tau\left(z_{n_{i}}\right)\right), \tau\left(z_{n_{i}}^{\prime}\right)\right) \rightarrow 0$ by uniform continuity of $\tau$ on $S$.

By property (H3) of holonomies, $d_{C^{r}}\left(H_{x, y}^{s}\right.$, Id $) \leq c d_{X}(x, y)^{\beta \rho}$, where $c$ is independent of $x$ and $y \in W_{\mathrm{loc}}^{s}(x)$. Hence

$$
\left\|D_{\mathcal{A}_{x}^{n}(t)} H_{f^{n} x, f^{n} y}^{s}-\mathrm{Id}\right\| \leq \kappa d_{C^{r}}\left(H_{f^{n} x, f^{n} y}^{s}, \mathrm{Id}\right) \leq \kappa c d_{X}\left(x_{n}, y_{n}\right)^{\beta \rho} \rightarrow 0 \text { as } n \rightarrow \infty .
$$

Using Lemma 4.3 and boundedness of $\tau$ on $S$, we conclude that the last term in (4.3) tends to 0 as $n \rightarrow \infty$. Therefore, $d\left(\tau\left(z^{\prime}\right),\left(D_{t} H_{x y}^{s}\right)_{*}(\tau(z))\right)=0$.

4.5. Hölder continuity of $\hat{\tau}$. Now we consider a particular measure on $X \times \mathcal{M}$. Let $\mu$ be the measure of maximal entropy for $(X, f)$. For each $x \in X$, we have the normalized volume $m_{x}$ on the fiber $\mathcal{M}_{x}$ induced by the metric $\hat{\tau}$, which is Hölder continuous on $\mathcal{M}_{x}$. We consider the measure $\hat{\mu}$ on $X \times \mathcal{M}$ given by $\hat{\mu}=\int m_{x} d \mu(x)$, that is, for any Borel measurable set $S \subset X \times \mathcal{M}$,

$$
\hat{\mu}(S)=\int_{X} m_{x}\left(S \cap \mathcal{M}_{x}\right) d \mu(x) .
$$


Clearly, the measure $\hat{\mu}$ is $F$-invariant. While it is not necessarily ergodic, we can consider its ergodic components and the corresponding partition $\xi$ of $X \times \mathcal{M}$. The Hopf argument yields that, up to a set of measure zero, every local stable set is contained in an element of $\xi$. So we can apply Proposition 4.4 to ergodic components of $\hat{\mu}$ we obtain the following.

Corollary 4.5. There exists a set $\hat{G} \subset X \times \mathcal{M}$ with $\hat{\mu}(\hat{G})=1$ such that $\hat{\tau}$ on $\hat{G}$ is invariant under the holonomies, that is,

$$
\hat{\tau}\left(z^{\prime}\right)=\left(D_{t} H_{x, y}^{s}\right)_{*}(\hat{\tau}(z)) \text { for all } z=(x, t) \in \hat{G} \text { and all } z^{\prime}=\left(y, t^{\prime}\right) \in \hat{G} \cap \tilde{W}_{l o c}^{s}(z) \text {. }
$$

Now we establish $\mu$-essential invariance of $\hat{\tau}_{x}$, as a Riemannian metric on the whole fiber $\mathcal{M}_{x}$, under the stable and unstable holonomies of the cocycle $\mathcal{A}$ over $X$.

Proposition 4.6. There exists a set $G \subset X$ with $\mu(G)=1$ such that or any $x, y, y^{\prime} \in$ $G$ with $y \in W_{l o c}^{s}(x)$ and $y^{\prime} \in W_{l o c}^{s}(x)$, the diffeomorphisms

$$
H_{x, y}^{s}:\left(\mathcal{M}_{x}, \hat{\tau}_{x}\right) \rightarrow\left(\mathcal{M}_{y}, \hat{\tau}_{y}\right) \text { and } H_{x, y^{\prime}}^{u}:\left(\mathcal{M}_{x}, \hat{\tau}_{x}\right) \rightarrow\left(\mathcal{M}_{y^{\prime}}, \hat{\tau}_{y^{\prime}}\right) \text { are isometries. }
$$

Proof. We will obtain a set $G^{s}$ of full measure for the stable holonomies. A similar argument gives a full measure set $G^{u}$ for the unstable holonomies, and $G=G^{s} \cap G^{u}$.

Let $\hat{G} \subset X \times \mathcal{M}$ be as in Corollary 4.5 and let $G^{s}=\pi(\hat{G}) \subset X$, where $\pi$ is the projection from $X \times \mathcal{M}$ to $X$. Then we have $\mu\left(G^{s}\right)=1$ and $m_{x}\left(\mathcal{M}_{x} \cap \hat{G}\right)=1$ for $\mu$ almost all $x \in G^{s}$. Discarding a set of measure zero, we can assume that $\hat{G}$ is $F$-invariant and

$$
m_{x}\left(\mathcal{M}_{x} \cap \hat{G}\right)=1 \text { for all } x \in G^{s} .
$$

Now we show that if $x, y \in G^{s}$ and $y \in W_{l o c}^{s}(x)$, then $H_{x, y}^{s}$ is an isometry between the fibers $\left(\mathcal{M}_{x}, \hat{\tau}_{x}\right)$ and $\left(\mathcal{M}_{y}, \hat{\tau}_{y}\right)$. Since $H_{x, y}^{s}: \mathcal{M}_{x} \rightarrow \mathcal{M}_{y}$ is a diffeomorphism, it maps sets of zero volume to sets of zero volume. The set

$$
E=\left(\mathcal{M}_{x} \cap \hat{G}\right) \cap\left(\left(H_{x, y}^{s}\right)^{-1}\left(\mathcal{M}_{y} \cap \hat{G}\right)\right)
$$

satisfies $E \subset \hat{G}, H_{x, y}^{s}(E) \subset \hat{G}$, and $m_{x}(E)=1$, and in particular $E$ is dense in $\mathcal{M}_{x}$. By Corollary 4.5 we have

$$
\hat{\tau}\left(z^{\prime}\right)=\left(D_{t} H_{x, y}^{s}\right)_{*}(\hat{\tau}(z)) \text { for all } z \in E .
$$

Thus $D H_{x, y}^{s}$ is isometric on the dense set $E$ and, as the Riemannian metrics $\hat{\tau}_{x}$ and $\hat{\tau}_{y}$ are $\alpha$-Hölder continuous along the fibers, we conclude that the diffeomorphism

$$
H_{x, y}^{s}:\left(\mathcal{M}_{x}, \hat{\tau}_{x}\right) \rightarrow\left(\mathcal{M}_{y}, \hat{\tau}_{y}\right) \text { is an isometry for all } x, y \in G^{s} \text { with } y \in W_{l o c}^{s}(x) .
$$

We denote by $\mathcal{T}(\mathcal{M}, \alpha)$ the space of $\alpha$-Hölder continuous Riemannian metrics on $\mathcal{M}$ equipped with $C^{\alpha}$ distance $d_{\mathcal{T} \alpha}$. Then the $\mu$-essential invariance of $\hat{\tau}$ yields $\mu$ essential $\beta \rho$-Hölder continuity of $\hat{\tau}$ as a function from $X$ to $\mathcal{T}(\mathcal{M}, \alpha)$ along the stable and unstable leaves in $X$. 
Corollary 4.7. The function $x \mapsto \hat{\tau}_{x}$ is $\beta \rho$-Hölder continuous on $G$ along the stable and unstable leaves in $X$ as a function from $X$ to $\mathcal{T}(\mathcal{M}, \alpha)$, that is

$$
d_{\mathcal{T} \alpha}\left(\hat{\tau}_{x}, \hat{\tau}_{y}\right) \leq C d_{X}(x, y)^{\beta \rho} \quad \text { for all } x, y \in G \text { with } y \in W_{l o c}^{s / u}(x) .
$$

Proof. By Proposition 3.3 (H3) the holonomies $H^{s / u}$ are $\beta \rho$-Hölder continuous in $\operatorname{Diff}^{r}(\mathcal{M}), r=1+\alpha$. By Proposition 4.6, $H_{x, y}^{s / u}\left(\hat{\tau}_{x}\right)=\hat{\tau}_{y}$ for all $x, y \in G$ with $y \in W_{l o c}^{s / u}(x)$. Now using Lemma 4.3 and boundedness of $\hat{\tau}$, we obtain

$$
d_{\mathcal{T}}\left(\hat{\tau}_{x}, \hat{\tau}_{y}\right) \leq c_{1} d_{C^{r}}\left(H_{x, y}^{s / u}, \mathrm{Id}\right) \leq c_{1} c d_{X}(x, y)^{\beta \rho} .
$$

Now the local product structure argument shows that $\hat{\tau}$ coincides $\mu$ almost everywhere with a $\beta \rho$-Hölder continuous stable and unstable holonomy invariant function $\tau: X \rightarrow \mathcal{T}(\mathcal{M}, \alpha)$. We consider a small open set $U$ in $X$ with the product structure of stable and unstable leaves, that is

$$
U=W_{l o c}^{s}\left(x_{0}\right) \times W_{l o c}^{u}\left(x_{0}\right) \stackrel{\text { def }}{=}\left\{W_{l o c}^{s}(x) \cap W_{l o c}^{u}(y) \mid x \in W_{l o c}^{s}\left(x_{0}\right), y \in W_{l o c}^{u}\left(x_{0}\right)\right\} .
$$

We recall that the measure of maximal entropy $\mu$ is equivalent to the product of its conditional measures on $W_{l o c}^{s}\left(x_{0}\right)$ and $W_{l o c}^{u}\left(x_{0}\right)$, which have full support on the corresponding leaves. Therefore for $\mu$ almost all local stable leaves in $U$, the set of points of $G$ on the leaf has full conditional measure, and hence full support. Without loss of generality, we can assume that $G$ has no points on the other leaves. Hence for any two points $x$ and $y$ in $G \cap U$ there exists a point $w \in W_{l o c}^{s}(x) \cap G$ such that $W_{l o c}^{u}(w) \cap W_{l o c}^{s}(y)$ is also in $G \cap U$. Then (4.4) and the local product structure of the stable and unstable manifolds yield that for all $x, y \in G \cap U$ we have

$$
d_{\mathcal{T}}(\hat{\tau}(x), \hat{\tau}(y)) \leq c_{3} d_{X}(x, y)^{\beta \rho} .
$$

Since this estimate holds for all $x, y \in G$, which is dense in $X, \hat{\tau}$ extends to a $\beta \rho$ Hölder continuous function $\tau: X \rightarrow \mathcal{T}(\mathcal{M}, \alpha)$, which is also invariant under the holonomies and the cocycle. As a function on $X \times \mathcal{M}, \tau$ is $\gamma$-Hölder continuous with $\gamma=\min \{\alpha, \beta \rho\}$. This completes the proof of Theorem 1.3.

\section{REFERENCES}

[AKL18] A. Avila, A. Kocsard, and X. Liu. Livšic theorem for diffeomorphism cocycles. To appear in GAFA.

[BK15] L. Backes and A. Kocsard. Cohomology of dominated diffeomorphism-valued cocycles over hyperbolic systems. Ergodic Theory Dynam. Systems, 36 (2015) 1703-1722.

[dlLO98] R. de la Llave and R. Obaya. Regularity of the composition operator in spaces of Hölder functions. Discrete and Continuous Dynamical Systems. 5 (1999), no. 1, 157-184.

[dlLW10] R. de la Llave and A. Windsor. Livšic theorem for non-commutative groups including groups of diffeomorphisms, and invariant geometric structures. Ergodic Theory Dynam. Systems, 30, no. 4 (2010), 1055-1100. 
[Gu] M. Guysinsky. Livšic theorem for cocycles with values in the group of diffeomorphisms. Preprint.

[H18] S. Hurtado. Examples of diffeomorphism group cocycles with no periodic approximation. To appear in Proceedings of the AMS. arXiv:1705.06361

[Ka11] B. Kalinin. Livšic theorem for matrix cocycles. Annals of Mathematics, 173 (2011), no. 2, 1025-1042.

[KP16] A. Kocsard and R. Potrie. Livšic theorem for low-dimensional diffeomorphism cocycles, Comment. Math. Helv. 91 (2016), 39-64.

[KaS10] B. Kalinin and V. Sadovskaya. Linear cocycles over hyperbolic systems and criteria of conformality. Journal of Modern Dynamics, vol. 4 (2010), no. 3, 419-441.

[KS13] B. Kalinin and V. Sadovskaya. Cocycles with one exponent over partially hyperbolic systems. Geometriae Dedicata, Vol. 167, Issue 1 (2013), 167-188.

$[\mathrm{KtH}]$ A. Katok and B. Hasselblatt. Introduction to the modern theory of dynamical systems. Encyclopedia of Mathematics and its Applications 54, Cambridge University Press, 1995.

[KtN] A. Katok and V. Nitica. Rigidity in Higher Rank Abelian Group Actions: Volume 1, Introduction and Cocycle Problem. Cambridge University Press, 2011.

[La] S. Lang. Fundamentals of Differential Geometry. New York: Springer-Verlag, 1999.

[Liv71] A. N. Livšic. Homology properties of Y-systems. Math. Zametki 10, 758-763, 1971.

[Liv72] A. N. Livšic. Cohomology of dynamical systems. Math. USSR Izvestija 6, 1278-1301, 1972.

[NT95] V. Nitica and A. Török. Cohomology of dynamical systems and rigidity of partially hyperbolic actions of higher-rank lattices. Duke Math. J., 79(3), 1995, 751-810.

[NT96] V. Nitica and A. Török. Regularity results for the solutions of the Livsic cohomology equation with values in diffeomorphism groups. Ergodic Theory Dynam. Systems 16 (1996), no. 2, 325333.

[NT98] V. Nitica and A. Török. Regularity of the transfer map for cohomologous cocycles. Ergodic Theory Dynam. Systems, 18(5), 1998, 1187-1209.

[S05] V. Sadovskaya. On uniformly quasiconformal Anosov systems. Math. Res. Lett., vol. 12 (2005), no. 3, 425-441.

[S15] V. Sadovskaya. Cohomology of fiber bunched cocycles over hyperbolic systems. Ergodic Theory Dynam. Systems, Vol. 35, Issue 8 (2015), 2669-2688.

[PW01] M. Pollicott and C. P. Walkden. Livšic theorems for connected Lie groups. Trans. Amer. Math. Soc., 353(7), 2001, 2879-2895.

[Sch99] K. Schmidt. Remarks on Livšic theory for non-Abelian cocycles. Ergodic Theory Dynam. Systems, 19(3), 1999, 703-721.

[Schr98] S. J. Schreiber. On growth rates of subadditive functions for semi-flows. J. Differential Equations, 148 (1998), 334-350.

[T06] M. Taylor. Existence and Regularity of Isometries. Trans. Amer. Math. Soc., Vol. 358, No. 6 (2006), 2415-2423.

Department of Mathematics, The Pennsylvania State University, University Park, PA 16802, USA.

E-mail address: sadovskaya@psu.edu 